\title{
Baryon light-cone wave functions in chiral quark-soliton model
}

\author{
Cédric LORCÉ* \\ University of Liège, Belgium \\ E-mail: C. lorce@ulg.ac.be
}

\begin{abstract}
We used the recent formulation of Chiral Quark-Soliton Model on the light cone to study static baryon properties by means of light-cone baryon wave functions. This model considers light baryons as made of quarks bound by a relativistic mean pion field with a non-trivial topology. The expression for light-cone wave functions incorporates chiral symmetry and special relativity, and therefore naturally the whole quark-antiquark sea plus quark orbital angular momentum. Using these light-cone wave functions we have computed many observables, such as vector, axial and tensor charges, and magnetic moments for octet, decuplet and even hypothetic antidecuplet. Our results are compared with the available experimental data.
\end{abstract}

LIGHT CONE 2008 Relativistic Nuclear and Particle Physics

July 7-11, 2008

Mulhouse, France

\footnotetext{
* Speaker.
} 


\section{Introduction}

Light-cone wave functions provide a very powerful tool to study the nucleon structure. While usually these wave functions are chosen rather arbitrarily, e.g. harmonic oscillator solutions, the Chiral Quark-Soliton Model provide us with a consistent wave function derived from an effective Lagrangian.

\section{Relativistic Mean Field Approximation}

Chiral Quark-Soliton Model ( $\chi$ QSM) is based on the following Lagrangian

$$
\mathscr{L}_{\chi \mathrm{QSM}}=\bar{\psi}\left(i \gamma^{\mu} \partial_{\mu}-M U^{\gamma_{5}}\right) \psi
$$

where $U^{\gamma_{5}}$ is a matrix incorporating the pion field. In the large $N_{C}$ limit, the pion field can be considered as a solitonic mean field. The spectrum of this model consists of a discrete level and a whole Dirac sea. The position of the discrete level and the distortion of the Dirac sea are directly related to the size of the soliton. The soliton profile is obtained self-consitently by requiring minimization of the nucleon mass.

One can write an expression for the baryon wave function

$$
\left|\Psi_{B}\right\rangle=\left[\prod_{i=1}^{N_{C}} \int\left(\mathrm{d} \mathbf{p}_{i}\right) F\left(\mathbf{p}_{i}\right) a^{\dagger}\left(\mathbf{p}_{i}\right)\right] \exp \left(\int(\mathrm{d} \mathbf{p})\left(\mathrm{d} \mathbf{p}^{\prime}\right) a^{\dagger}(\mathbf{p}) W\left(\mathbf{p}, \mathbf{p}^{\prime}\right) b^{\dagger}\left(\mathbf{p}^{\prime}\right)\right)\left|\Omega_{0}\right\rangle,
$$

where the first factor corresponds to filling in the discrete level with $N_{C}$ quarks and the second one is the coherent exponential describing the whole Dirac sea. The function $F(\mathbf{p})$ represents the wave function of a discrete-level quark and $W\left(\mathbf{p}, \mathbf{p}^{\prime}\right)$ the wave function of a quark-antiquark pair. These are obtained by solving the one-particle Hamiltonian and quark propagator in the soliton background.

Since the model is relativistically invariant, (2.1) can be translated to the infinite momentum frame (IMF). In this particular frame, $W$ is a function of the fractions of the baryon longitudinal momentum carried by the quark $z$ and antiquark $z^{\prime}$ of the pair and their transverse momenta $\mathbf{p}_{\perp}, \mathbf{p}_{\perp}^{\prime}$

$$
\begin{aligned}
W_{j^{\prime} \sigma^{\prime}}^{j \sigma}\left(z, \mathbf{p}_{\perp} ; z^{\prime}, \mathbf{p}_{\perp}^{\prime}\right)= & \frac{M \mathscr{M}}{2 \pi Z}\left\{\Sigma_{j^{\prime}}^{j}(\mathbf{q})\left[M\left(z^{\prime}-z\right) \tau_{3}+\mathbf{Q}_{\perp} \cdot \tau_{\perp}\right]_{\sigma^{\prime}}^{\sigma}\right. \\
& \left.-i \Pi_{j^{\prime}}^{j}(\mathbf{q})\left[-M\left(z^{\prime}+z\right) \mathbf{1}+i \mathbf{Q}_{\perp} \times \tau_{\perp}\right]_{\sigma^{\prime}}^{\sigma}\right\},
\end{aligned}
$$

where $\mathbf{q}=\left(\left(\mathbf{p}+\mathbf{p}^{\prime}\right)_{\perp},\left(z+z^{\prime}\right) \mathscr{M}\right)$ is the three-momentum of the pair as a whole transferred from the background fields $\Sigma(\mathbf{q})$ and $\Pi(\mathbf{q}), \tau_{1,2,3}$ are Pauli matrices, $\mathscr{M}$ is the baryon mass and $M$ is the constituent quark mass. In order to simplify the notations we used

$$
Z=\mathscr{M}^{2} z z^{\prime}\left(z+z^{\prime}\right)+z\left(\mathbf{p}_{\perp}^{\prime 2}+M^{2}\right)+z^{\prime}\left(\mathbf{p}_{\perp}^{2}+M^{2}\right), \quad \mathbf{Q}_{\perp}=z \mathbf{p}_{\perp}^{\prime}-z^{\prime} \mathbf{p}_{\perp} .
$$

The discrete-level wave function in IMF is given by

$$
F^{j \sigma}\left(z, \mathbf{p}_{\perp}\right)=\sqrt{\frac{\mathscr{M}}{2 \pi}}\left[\varepsilon^{j \sigma} h(p)+\left(p_{z} \mathbf{1}+i \mathbf{p}_{\perp} \times \tau_{\perp}\right)_{\sigma^{\prime}}^{\sigma} \varepsilon^{j \sigma^{\prime}} \frac{j(p)}{|\mathbf{p}|}\right]_{p_{z}=z \mathscr{M}-E_{l e v}},
$$




\begin{tabular}{|c|ccc|}
\hline & $3 \mathrm{Q}$ & $5 \mathrm{Q}$ & $7 \mathrm{Q}$ \\
\hline$B_{\mathbf{8}} \& B_{\mathbf{1 0}}$ & $\approx 72 \%$ & $\approx 21 \%$ & $\approx 7 \%$ \\
$B_{\overline{\mathbf{1 0}}}$ & $0 \%$ & $\approx 61 \%$ & $\approx 39 \%$ \\
\hline
\end{tabular}

Table 1: Baryon composition up to the 7Q contribution.

where $h$ and $j$ are the upper and lower components of the Dirac spinor, respectively, and $E_{l e v}=200$ $\mathrm{MeV}$. They are obtained self-consistently for the quark mass $M=345 \mathrm{MeV}$. For more details, see $[1,2]$.

The baryon light-cone wave function (2.1) is expanded in Fock space and allows one to compute individually the contribution of each Fock component. Charges are simply obtained by considering the matrix element of the corresponding quark current between the Fock components of the baryon. Using the Drell-Yan-West frame, only the diagonal transitions in Fock space will contribute, i.e. $n$ quarks into $n^{\prime}$ quarks with $n=n^{\prime}$. Note also that (2.1) is a generic formula for all members of the lowest octet, decuplet and hypothetic antidecuplet. In actual computation one performs a rotation of (2.1) in ordinary and flavor spaces, and then projects it onto the quantum numbers of a given baryon.

\section{Results}

In this contribution to the proceedings, we present only a selected part of our results. The complete list can be found in [2] and further papers in preparation.

\subsection{Baryon Components}

Since (2.1) incorporates the whole tower of Fock components, one can estimate which fraction of the baryon is actually made of $n$ quarks $(n \mathrm{Q})$. Table 1 shows the composition of light baryons in terms of Fock components up to $7 \mathrm{Q}$. Concerning ordinary baryons, our estimate for the $5 \mathrm{Q}$ component is consistent with other approaches. What is interesting is the estimation of the $7 \mathrm{Q}$ contribution. As expected, it is rather small but might be non-negligible. For exotic baryons, there cannot be any $3 \mathrm{Q}$ contribution. Note however that the dominant contribution (5Q) is smaller than the dominant one $(3 \mathrm{Q})$ in ordinary baryons. One can therefore expect exotic baryons to be more sensible to the Dirac sea.

\subsection{Angular Momentum}

$\chi$ QSM is a relativistic model. Quark wave function has therefore both $L=0(h)$ and $L=1$ $(j)$ contributions. Due to the latter one, vector, axial and tensor currents do not see the same quark distribution. We should not therefore expect axial and tensor charges to be the same, as predicted by the non-relativistic Naive Quark Model (NQM). Like other relativistic models, $\chi$ QSM suggests the following pattern in magnitudes:

$$
|q|>|\delta q|>|\Delta q|
$$




\begin{tabular}{|c|ccc|}
\hline & $g_{A}^{(3)}$ & $g_{A}^{(8)}$ & $g_{A}^{(0)}$ \\
\hline NR 3Q & 1.667 & 0.577 & 1 \\
3Q & 1.435 & 0.497 & 0.861 \\
3Q+5Q & 1.241 & 0.444 & 0.787 \\
Exp. & $1.257 \pm 0.003$ & $0.34 \pm 0.02$ & $0.31 \pm 0.07$ \\
\hline
\end{tabular}

Table 2: Axial charges of the proton.

Indeed, a vector probe will see the following quark density

$$
h^{2}+2 \frac{p_{z}}{|\mathbf{p}|} h j+\frac{p_{z}^{2}+\mathbf{p}_{\perp}^{2}}{\mathbf{p}^{2}} j^{2}
$$

while an axial probe will see

$$
h^{2}+2 \frac{p_{z}}{|\mathbf{p}|} h j+\frac{p_{z}^{2}-\mathbf{p}_{\perp}^{2}}{\mathbf{p}^{2}} j^{2}
$$

and a tensor probe will see

$$
h^{2}+2 \frac{p_{z}}{|\mathbf{p}|} h j+\frac{p_{z}^{2}}{\mathbf{p}^{2}} j^{2} .
$$

Note that if we keep the last term only, we recover the Melosh rotation. However, it is known that a completely covariant picture asks for both $h$ and $j$ [3]. The first two terms are not negligible and one can expect that the correct relativistic effect due to orbital angular momentum is smaller than the one obtained through Melosh rotation.

\subsection{Axial Charges}

Table 2 shows the axial charges of the proton. The first line corresponds to the non-relativistic limit (3Q only in $L=0$ state) and coincides exactly with NQM, as it should be. The second line corresponds to the case where there are three relativistic quarks. The change is therefore due to the orbital angular momentum of the quarks. Note that as expected, the effect is smaller than the one obtained by pure Melosh rotation. The third line incorporates the correction obtained when one adds an extra quark-antiquark pair to the picture. Both quark orbital angular momentum and quarkantiquark pairs bring the axial charges closer to experimental values (fourth line). The discrepancy observed mainly for $g_{A}^{(0)}$ could in principle be attributed to the fact that the present approach is based on flavor $S U(3)$ symmetry [4]. The model scale is $Q_{0}^{2}=0.36 \mathrm{GeV}^{2}$.

\subsection{Proton Flavor Content}

Since we have explicit $n \mathrm{Q}$ light-cone wave functions, it is rather easy to separate the contribution of individual flavors. Moreover, it is also possible to separate the discrete-level, sea quark and antiquark contributions. Table 3 shows the different contributions to the proton vector content up to the 7Q contribution. The first column contains the discrete-level contribution, while the next two columns contain the sea contribution that arise from the non-leading Fock components. 


\begin{tabular}{|c|cccc|}
\hline & $q_{\text {val }}$ & $q_{s}$ & $\bar{q}$ & $q_{\text {tot }}$ \\
\hline $\mathrm{u}$ & 1.923 & 0.202 & 0.125 & 2 \\
$\mathrm{~d}$ & 1.017 & 0.128 & 0.145 & 1 \\
$\mathrm{~s}$ & 0.060 & 0.028 & 0.088 & 0 \\
\hline
\end{tabular}

Table 3: Vector content of the proton up to $7 \mathrm{Q}$.

\begin{tabular}{|c|ccc|}
\hline & $\mu_{p} / \mu_{n}$ & $\mu_{p}\left(\mu_{N}\right)$ & $\mu_{n}\left(\mu_{N}\right)$ \\
\hline 3Q & -1.5 & 2.534 & -1.689 \\
3Q+5Q & -1.479 & 2.900 & -1.961 \\
Exp. & -1.460 & 2.793 & -1.913 \\
\hline
\end{tabular}

Table 4: Proton and neutron magnetic moments.

The last column gives the effective number of quark $q_{v a l}+q_{s}-\bar{q}$, or valence quarks. One can identify valence and discrete-level quarks only in a perturbative picture (gluon splitting) of the sea where $q_{s}=\bar{q}$. In a non-perturbative picture, the flavor content of the discrete level is perturbed (baryon-meson fluctuation).

Let us note two remarkable features of the non-perturbative picture. First, we obtain an isospin-asymmetric sea leading to the violation of Gottfried sum rule [5]. However, the model does not reproduce quantitatively this violation $\bar{d}-\bar{u}=0.019$ ( $\chi Q S M)$ v.s. $0.118 \pm 0.012$ (Exp.) [6]. Second, since the discrete level contains strangeness, one should expect to observe an asymmetric strange quark distribution $s(z)-\bar{s}(z) \neq 0$. Another consequence of this would be a non-zero strangeness contribution to tensor charges, the latter being only sensitive to discrete-level contribution.

\subsection{Nucleon Magnetic Moments}

One of the most striking successes of this approach concerns the evaluation of magnetic moments. Table 4 shows our values for the proton and magnetic moments compared to experimental data. First of all, one can notice the nice matching. We would like to emphasize that the technique used is a totally $a b$ initio computation and does not therefore contain a fitting procedure to experimental values. Note also that the $5 \mathrm{Q}$ contribution is important. This means that the sea contributes significantly to the nucleon magnetic moments.

\section{6 $\gamma N \rightarrow \Delta$ Transition and Baryon Shape}

One cannot observe any quadrupole distortion of a spin-1/2 particle. Such a distortion can however be observed in the electromagnetic transition between nucleon and $\Delta$. Table 5 shows our values for the $\gamma N \rightarrow \Delta$ transition observables compared to experimental data. A non-vanishing negative value for $G_{E}^{*}$ indicates that the system is oblate. We have also shown explicitly that $G_{E}^{*}$ is directly proportional to the quadrupolar moment generated by the sea. 


\begin{tabular}{|c|ccc|}
\hline & $3 \mathrm{Q}$ & $3 \mathrm{Q}+5 \mathrm{Q}$ & Exp. \\
\hline$A_{3 / 2}\left(\mathrm{GeV}^{-1 / 2}\right)$ & -0.296 & -0.232 & $-0.250 \pm 0.008$ \\
$A_{1 / 2}\left(\mathrm{GeV}^{-1 / 2}\right)$ & -0.171 & -0.129 & $-0.135 \pm 0.006$ \\
$G_{M}^{*}\left(\mu_{N}\right)$ & 2.389 & 2.820 & 2.798 \\
$G_{E}^{*}\left(\mu_{N}\right)$ & 0 & 0.026 & 0.046 \\
$R_{E M}=-\frac{G_{E}^{*}}{G_{M}^{*}}$ & 0 & $-0.9 \%$ & $-1.6 \%$ \\
$\Gamma_{p \Delta}(\mathrm{MeV})$ & 0.411 & 0.573 & 0.564 \\
\hline
\end{tabular}

Table 5: $\gamma N \rightarrow \Delta$ transition observables.

\section{Acknowledgments}

The author is grateful to RUB TP2 for its kind hospitality, to D. Diakonov and M. Polyakov for their comments and suggestions. The author is also indebted to J. Cugnon for his help and advices. The author would also like to express his gratitude towards LC2008 organizers for the very nice and interesting workshop. This work has been supported by the National Funds of Scientific Research, Belgium.

\section{References}

[1] V. Petrov and M. Polyakov, Light cone nucleon wave function in the quark-soliton model, hep-ph/0307077.

D. Diakonov and V. Petrov, Estimate of the Theta+ width in the Relativistic Mean Field Approximation, Phys. Rev. D72 (2005) 074009 [hep-ph/ 0505201$].$

[2] C. Lorcé, Improvement of the Theta+ width estimation method on the Light Cone, Phys. Rev. D74 (2006) 054019 [hep-ph/0603231].

C. Lorcé, Baryon vector and axial content up to the 7Q component, Phys. Rev. D78 (2008) 034001 [hep-ph/0708.3139].

C. Lorcé, Tensor charges of light baryons in the Infinite Momentum Frame, hep-ph/arXiv:0708.4168.

[3] S.J. Brodsky, J.R Hiller, D.S. Hwang and V.A. Karmanov, The covariant structure of light-front wave functions and the behavior of hadronic form factors, Phys. Rev. D69 (2004) 076001 [hep-ph/0311218].

[4] H.C. Kim, M. Praszalowicz and K. Goeke, Spin structure of the octet baryons, Acta Phys. Pol. B32 (2001) 1343 [hep-ph/0007022].

[5] K. Gottfried, Sum Rule for High-Energy Electron-Proton Scattering, Phys. Rev. Lett. 18 (1967) 1174.

[6] R.S. Towell et al. [FNAL E866/NuSea Coll.], Improved Measurement of the $\bar{d} / \bar{u}$ Asymmetry in the Nucleon Sea, Phys. Rev. D64 (2001) 052002 [hep-ex/ 0103030 ]. 